\title{
EL CONCEPTO DE ANISOMORFISMOS CULTURAL EN EL ÁMBITO JURÍDICO: CONCEPTO Y APLICACIONES TRADUCTOLÓGICAS SOBRE LA LEGISLACIÓN ESPAÑOLA EN COMPARACIÓN CON LA MARROQUÍ Hasna Karim
}

Campus Universitaire Privé de Marrakech - CUPM (Marrakech, Marruecos)

\section{ABSTRACT}

In this article, we want to emphasize the relevance of the documentation process to solve problems related to the translation of cultural anysomorphisms in Legal Texts (Arabic-Spanish / Spanish-Arabic).

KEYWORDS: Cultural anysomorphisms, Translation of Cultural anysomorphisms, Legal Translation, Translation Arabic-Spanish, Translation Spanish-Arabic.

\section{RESUMEN}

En este artículo pretendemos hacer hincapié en la relevancia de la documentación para resolver problemas relacionados con la traducción de anisomorfismos culturales en textos jurídicos (árabe-español / español-árabe).

PALABRAS CLAVE: Anisomorfismos culturales, traducción de anisomorfismos culturales, traducción jurídica, traducción árabe-español, traducción español-árabe.

\section{INTRODUCCIÓN}

La traducción de los anisomorfismos plantea cierto grado de dificultad y exige la aplicación de distintos tipos de "estrategias" o "soluciones" según los casos. La documentación y la terminología pueden ser herramientas muy útiles para aclarar ambigüedades y confusiones que imponen estos anisomorfismos.

De manera que, vamos a poner de relieve en el presente artículo la importante labor de la documentación y la terminología en la traducción especializada, hasta tal punto que no se puede realizar una traducción especializada sin términos sintéticos. Sin embargo, a la hora de extraer terminología, no se puede recurrir a la traducción como método o fuente, 
excepto en casos de creación de términos para lenguas sin recursos especializados. Entonces se trata de un concepto con carácter unilateral.

Sin embargo, pretendemos demostrar en este capítulo que la relación existente entre terminología y la documentación es de carácter bilateral, de forma que, el trabajo terminológico no puede llevarse a cabo sin documentación, y una parte del trabajo documental no se puede realizar sin terminología, tomando en consideración el desarrollo de las tecnologías de la información y la documentación.

\section{EL ANISOMORFISMO}

El término anisomorfismo está vinculado a un concepto muy usado en el campo de la traducción y la traductología: la intraducibilidad.

Anisomorfismo es lo opuesto a isomorfismo, término éste muy querido durante mucho tiempo en los estudios de lingüística, sobre todo durante el auge del estructuralismo. Isomorfismo quiere decir simetría o paralelismo entre dos sistemas lingüísticos; consecuentemente, anisomorfismo significa asimetría. Los estudios teóricos de traducción han puesto de relieve que la transferencia lingüística desde la lengua de partida a la de llegada está siempre condicionada por una serie de asimetrías o anisomorfismos.

\section{CLASES DE ANISOMORFISMOS}

En este momento, convendría preguntarse por qué esa traducción idéntica no es posible, por qué las lenguas son esencialmente asimétricas o anisomórficas con el fin de aclarar el malentendido en que se ha basado gran parte de la teoría de la traducción durante siglos. Pues bien, existen al menos cuatro grandes asimetrías o anisomorfismos que hacen ontológicamente imposible que un texto traducido pueda ser idéntico a su original: lingüístico; cultural; pragmático; interpretativo, aunque aquí sólo comentaremos los aspectos lingüístico y cultural.

El anisomorfismo lingüístico se basa en que las lenguas no son correlatos objetivos de la realidad, sino taxonomías, intentos de separar en categorías lo que en realidad es un continuum sin barreras.

En cualquier otra variedad lingüística, se puede percibir el anisomorfismo lingüístico, ya que todas las lenguas son, por definición sistemáticas, pero también asimétricas, es decir, tienen excepciones. La misma palabra "Derecho" es un claro ejemplo de anisomorfismo léxico, puesto que no existe un adjetivo para esta palabra, esto es, no se puede decir (derechal). Y lo mismo le ocurre al adjetivo "jurídico", pero esta vez es a la inversa, dado que 
carece de sustantivo. Por esta razón, el nombre "Derecho" y el adjetivo "jurídico" han formado un buen matrimonio de conveniencia léxica, uno como sustantivo y otro como adjetivo.

El anisomorfismo cultural es el más difícil de abordar, porque la cultura está formada por una serie de pautas, creencias, costumbres, ideologías, presuposiciones, etc. que con el paso del tiempo una comunidad acepta como naturales o propias del género humano.

\section{LA INTRADUCIBILIDAD, LA IDENTIDAD Y LA EQUIVALENCIA}

En realidad, muchos teóricos de la traducción consideran que la intraducibilidad no es posible en traducción, ya que nunca serán idénticos el texto de la lengua de partida y el texto de la lengua de llegada. No aspiramos a que sean idénticos, lo que nos proponemos es que sean equivalentes. Siempre buscamos la equivalencia y no la identidad.

Lo que estos autores consideran "equivalentes" son unidades de sentido en el plano abstracto del sistema, con lo que parecen reducir la traducción a una sustitución terminológica, como muestra su definición de la equivalencia como procedimiento de transferencia: "Procédé de traduction qui rend compte de la même situation que dans l'original, en ayant recours à une redaction entièrement differente" (Vinay \& Darbelnet, 1977:8-9)1.

Aunque no sea posible la traducción exacta en sus términos más estrictos, sabemos que desde tiempos inmemoriales nos comunicamos a través de traducciones, y aquí queremos resaltar la palabra comunicación, que es una de las que da más sentido a la traducción.

Por otra parte, también sabemos que los organismos internacionales pueden funcionar gracias a las versiones de unas lenguas a otras que hacen sus aplicados y profesionales traductores, aunque, en estos casos, muchas veces se trabaja con una cultura jurídica supranacional - de referencia, lo que hace mucho más fácil la búsqueda y/o adopción de equivalentes en las distintas versiones lingüísticas.

\section{SOBRE EL TEMA OBJETO DE ESTUDIO}

No vamos a entrar aquí en el análisis de casos concretos, sino en la delimitación del problema objeto de estudio. El tratamiento de los 
anisomorfismos en la traducción de "legislación", en este caso marroquí y española.

El problema que nos plantea la traducción de legislación "nacional", no pensada para surtir efecto fuera de las fronteras de un Estado, es precisamente su "categorización" dentro de un sistema de creencias, costumbres, presupuestos, etc. $\mathrm{O}$ si se prefiere, toda legislación nacional presupone una "visión del mundo", por lo que no es trasladable, lingüísticamente hablando, de una lengua a otra, como si los universos culturales que las sustentan pudieran ser considerados como totalmente equivalentes o idénticos. A este respecto, tomando en consideración las culturas "marroquí" y "española", resulta evidente que la traducción de "anisomorfismos culturales" no resulta fácil y, en más de una ocasión, habrá de apoyarse en glosas intra o extratextuales para solucionar el problema de "incomunicación" o "intraducibilidad" existente entre ambas subculturas (jurídicas).

En cualquier caso, en función del tipo de traducción que se esté llevando a cabo (jurídica, jurada, judicial) y de la dirección en que se lleve a cabo (español-árabe o árabe-español), habrá que valorar cuál es la estrategia más adecuada para que la traducción sea considerada "aceptable" o, en muchos casos, sencillamente para que "surta efecto" en función de la finalidad que le haya sido atribuida y del destinatario de ésta.

Estas reflexiones teóricas previas sobre el problema de la traducción de anisomorfismos culturales en textos jurídicos ha sido objeto de consideración, in extenso, en la tesis doctoral de la autora, defendida en la Universidad de Málaga en marzo de 2010, y los resultados prácticos de la investigación llevada a cabo aparecerá publicada, a lo largo de 2011, en forma de glosarios bilingües (árabe-español / español-árabe) de unidades simples y complejas (términos y unidades fraseológicas) relativas al ámbito legislativo español y marroquí.

Por tanto, el objeto de este artículo no es otro que la reflexión en torno al problema que plantean los anisomorfismos culturales en la traducción jurídica, máxime cuando ésta se lleva a cabo entre dos culturas jurídicas tan cercanas en algunos aspectos, y tan alejadas en otros, como la española y la marroquí.

\section{BIBLIOGRAFÍA}

Alcaraz Varó, EnriQue y Brian Hughes, El español jurídico, Ariel Derecho, Barcelona, 2002.

Álvarez Calleja, María ANTONIA, Traducción jurídica (inglés- español), UNED, Madird, 2002. 
Alcina Caudet, Amparo y Silvia Gameró PÉREZ, La traducción científicatécnica y la terminología en la sociedad de la información, Universitat Jaume, Valencia, 2002.

Aránguez SÁnchez, Carlos y Esperana Alarcón Navío, Diccionario de términos jurídicos (francés-español/ español-francés), Comares, Granada, 2000.

Becker, J. Analecta, España y Marruecos, Pamplona, 2006.

Blanc, François Paul, Droit pénal général, Sochepress, Casablanca, 1984.

CAmargo HernánDeZ, César, Introducción al estudio del derecho penal: investigación técnica - jurídica del concepto del Derecho Penal, ensayo sobre metodología jurídicapenal, estudio sobre las fuentes del Derecho Penal, Bosh, D.L, Barcelona, 1964.

CARLIER, JEAN-YVES Y MiCHEL VER WILGHEN, Le statut personnel des musulmans. Droit comparé et droit international privé, Bruylant, Bruxelles, 1992.

Chafi, Mohammed, Vocabulaire Juridique et Politique. Marrakesh. Publications Universitaires du Maghreb, 1993.

CORnu, GÉrARD, linguistique juridique, Montchéstien, Paris, 2000.

CORnu, GÉRARD, Vocabulaire juridique, Presses Universitaires de France, Paris, 2000.

CORPAS PASTOR, GLORIA (ed.), Recursos documentasles y tecnológicos para la traducción del discurso jurídico (español, alemán, inglés, italiano, árabe), Editorial comares, Granada, 2003.

COVIÁN JUNCO, VÍCTOR, Código civil español con las correcciones y reformas introducidas posteriormente por la redacción de la Revista de los tribunales, Madrid, Góngora, 1956.

Cuello Contreras, JoAquín, El derecho penal español, Madrid, Dykinson, 2002

De Espalza, Mikel, Traducción del árabe, Gedisa, Barcelona, 2004.

Díez Ripoldés, José Luis, Derecho Penal Español Parte General en Esquemas, Editorial: Tirant lo Blanch, 2007.

Duro Moreno, Miguel, Introducción al Derecho inglés: la traducción jurídica inglésespañol en su entorno, Edisofer, Madrid, 2005.

FÉlix LEANDRO, ORTEGA ARJONILLA EMILIO (EDS.), Lecciones de teoría y práctica de la traducción, Universidad de Málaga, 1997.

GONZÁLEZ DE ANDÍA, MANUEL, Les principes généraux du droit de la nationalité en Espagne par comparaison avec ceux du droit Français, Paris, 1934.

Guastini, Ricardo, Estudio sobre la interpretación jurídica, Porúa, México, 2001.

HechaÏmé, CAmille, I., La traduction par les textes, Dar el-Macherek, Beirut, 1980.

Herzog, JACQuES, Le droit jurisprudentiel et le tribunal suprême en Espagne, Imprimerie F.Boisseau, Toulouse, 1942.

HuRTADO AlBIR, AMPARO, Traducción y traductología : Introducción a la traductología, Madrid, Cátedra, 2001.

J.P.VINAY, J.DARBELNET, Stylistique comparée du français et de l'anglais, Éditions Didier, Paris, 1958. 
LÓPEZ Guerra, Luis, El derecho español en el siglo XX, Madrid, Barcelona: Marcial Pons, Ediciones Jurídicas y Sociales, 2000.

MATTAR, ANTOINE, C., La traduction pratique: Français-arabe, arabe-français, Dar el Machreq, Beyrouth, 1987.

Mikel de EsPazA, Traducir del árabe, Gedisa, Baercelona, 2004.

Monzó NeBOT, ESTHER y ANABEL BORJA ALBI, La traducción y la interpretación en las relaciones jurídicas internacionales, Castellón. Universitat de Jaume I, 2005.

Mourad BoussetTA, Eléments de droit pénal général marocain, Université Cadi Ayyad, Marrakech, 2001-2002.

Muñoz Conde, Francisco, Código Penal, Edi. Ariel, 2006- 2007.

MuÑoz y Rivero, MARiano, Código penal español con todas las modificaciones en él introducidas hasta 1902, recopiladas por la redacción de la Revista de legislación Universal, Madrid, Liberias de Fé, 1902.

Newmark, Peter, Manual de traducción, Edi: 4, Cátedra, Madrid, 2004.

Ortega Arjonilla, Emilio y Elena Echeverría Pereda, Enseñanza de lenguas, traducción e interpretación (francés-español), Universidad de Málaga, 1996.

Ortega ArJonilla, EMILIO (DIR.): La traducción la interpretación jurídicas en la Unión Europea. Retos para la Europa de los ciudadanos. Editorial Comares, colección interlingua, Granada, 2008.

Popesco- Remniceano, Droit pénal en matière de devises. Études de droit comparé, Pédone, Paris, 1939.

RABADÁN, ROSA, Equivalencia y traducción: problemática de la equivalencia translémica inglés-español, Universidad de León, 1991.

RAIS MAÂTI, MARTIN DIDIER, Droit civil et commercial marocain, Société d'Édition et de diffusion Al Madariss, Casablanca, 1990.

SÂAD MOUmmI, Droit civil, Droit des obligations, édition El badii, Marrakech, 2000.

SAlES SALVADOR, DORA, La biblioteca de Babel: documentarse para traducir, Comares, Granada, 2005.

SÁNCHEZ- TEJERINA, IsAÍAS, La ley penal, el delito, la pena y otras medidas, Madrid: (S.N), 1950.

SAN Ginés Aguilar, PEDro y Emilio OrTEgA ArJONiLla (EDS.), Introducción a la traducción jurídica y jurada (francés- español) orientaciones metodológicas para la realización de traducción juradas y de documentos jurídicos, Comares, Granada, 1997.

SAN Ginés Aguilar, Pedro y Emilio OrTega ArJOnilla (EDS.), Introducción a la traducción juridica y jurada (inglés- español) orientaciones metodológicas para la realización de traducción juradas y de documentos juridicos, Comares, Granada, 1997.

SÉROUSSI, ROLAND, Introduction au droit comparé, Dunod, Paris, 2003. 
SERRANO FERNÁNDEZ, MARÍA, Estudio de derecho comparado sobre la interpretación de los contratos, Tirant lo Blanch, Valencia, 2005.

Velasco landecho, Carlos Mería, Concepción Molina BlázQuez, Derecho penal español. Parte especial: redactada conforme al nuevo Código Penal de 23 de noviembre de 1995, Madrid, Tecnos, 1996.

VV AA, Aproximación a los Estudios de traducción, Servicio de apoyo a la enseñanza, Universidad de Valladolid, 1997. 